\title{
IncRNA SNHG16 is associated with proliferation and poor prognosis of pediatric neuroblastoma
}

\author{
YONGBO YU ${ }^{1 *}$, FENG CHEN $^{2 *}$, YERAN YANG $^{1}$, YAQIONG JIN $^{1}$, JIN SHI $^{1}$, SHUJING HAN ${ }^{1}$, \\ PING CHU ${ }^{1}$, JIE LU $^{1}$, JUN TAI $^{3}$, SHENGCAI WANG ${ }^{3}$, WEI YANG ${ }^{4}$, \\ HUANMIN WANG ${ }^{4}$, YONGLI GUO ${ }^{1}$ and $\mathrm{XIN} \mathrm{NI}^{1,3}$ \\ ${ }^{1}$ Beijing Key Laboratory for Pediatric Diseases of Otolaryngology, Head and Neck Surgery, \\ MOE Key Laboratory of Major Diseases in Children, Beijing Pediatric Research Institute, \\ Departments of ${ }^{2}$ Functional Neurosurgery, ${ }^{3}$ Otolaryngology, Head and Neck Surgery \\ and ${ }^{4}$ Surgical Oncology, Beijing Children's Hospital, Capital Medical University, \\ National Center for Children's Health (NCCH), Beijing 100045, P.R. China
}

Received October 16, 2018; Accepted May 20, 2019

DOI: 10.3892/ijo.2019.4813

\begin{abstract}
Neuroblastoma (NB) is one of the most common extracranial solid tumors in children, which has complex molecular mechanisms. Increasing evidence has suggested that long noncoding RNAs (lncRNAs) account for NB pathogenesis. However, the function of small nucleolar RNA host gene 16 (SNHG16) in NB is currently unclear. In the present study, publically available data and clinical specimens were employed to verify the expression of SNHG16 in NB. Colony formation, real-time cell proliferation and migration assays were performed to demonstrate the status of cellular proliferation and migration. Flow cytometry was used to examine cell cycle progression in SH-SY5Y cells, and acridine orange/ethidium bromide staining and caspase-3/7 activity measurements were applied to study cell apoptosis. To explore the underlying mechanism of SNHG16 function, an online database was used to identify potential RNA-binding proteins that bind SNHG16. The expression of $S N H G 16$ was revealed to be in line with
\end{abstract}

Correspondence to: Professor Xin Ni, Department of Otolaryngology, Head and Neck Surgery, Beijing Children's Hospital, Capital Medical University, National Center for Children's Health (NCCH), 56 Nalishi Road, Beijing 100045, P.R. China

E-mail: nixin@bch.com.cn

Professor Yongli Guo, Beijing Key Laboratory for Pediatric Diseases of Otolaryngology, Head and Neck Surgery, MOE Key Laboratory of Major Diseases in Children, Beijing Pediatric Research Institute, Beijing Children's Hospital, Capital Medical University, National Center for Children's Health (NCCH), 56 Nalishi Road, Beijing 100045, P.R. China

E-mail: guoyongli@bch.com.cn

*Contributed equally

Key words: neuroblastoma, lncRNAs, SNHG16, proliferation the clinical staging of NB, and high $S N H G 16$ expression was positively associated with poor clinical outcome. Furthermore, SNHG16 silencing inhibited cell proliferation, repressed migration, and induced cell cycle arrest at the $G_{0} / G_{1}$ phase in SH-SY5Y cells. Additionally, apoptosis was undetectable in SH-SY5Y cells following SNHG16 silencing. Bioinformatics analysis revealed that $S N H G 16$ regulated cell proliferation in NB through transcriptional and translational pathways. These results suggested that $S N H G 16$ may serve important roles in the development and progression of NB, and could represent a potential target for NB therapy.

\section{Introduction}

Neuroblastoma (NB) is one of the most common extracranial solid tumors in children, accounting for $15 \%$ of all childhood deaths from cancer (1). It is almost exclusively a pediatric malignancy, and $>90 \%$ of patients are diagnosed at $<10$ years of age $(2,3)$. NB originates in neural crest cells of the sympathetic nervous system, which are mainly found in the adrenal gland, neck, chest, abdomen and pelvic cavity. Although the clinical diagnosis and treatment for NB are continually improving, the 5-year survival rate for children with high-risk NB remains $<50 \%$ (4).

The mechanisms underlying NB pathogenesis and development are complex, involving genetic and epigenetic alterations, chromosomal changes, and the altered expression of microRNAs (miRNAs/miRs) and long non-coding RNAs (lncRNAs) $(5,6)$. Among these genetic changes, the Children's Oncology Group revealed that MYCN proto-oncogene, bHLH transcription factor $(M Y C N)$ amplification is an independent marker for NB prognosis and risk stratification $(7,8)$. However, MYCN amplification occurs in only $20-30 \%$ of patients with primary NB (9). Due to the diversity of clinical phenotypes and complex biological characteristics, there is an urgent need to identify precise biological markers for NB diagnosis and prognosis, as well as potential molecular targets for chemotherapy. 
lncRNAs are non-coding RNA transcripts $>200$ nucleotides long, which are involved in transcriptional and post-transcriptional regulation (10), and have important value for the diagnosis and treatment of tumors. They contribute to various biological processes in tumorigenesis, including tumor proliferation, metastasis, differentiation and cell death. IncRNAs can be detected in urine, and prostate cancer-associated 3 was recently approved by the US Food and Drug Administration to identify prostate cancer $(11,12)$. In pediatric NB, various lncRNAs, including metastasis-associated lung adenocarcinoma transcript 1 (MALAT1), HOXD antisense growth-associated long non-coding RNA and miR-100-let-7a-2-miR-125b-1 cluster host gene (linc-NeD125), are involved in differentiation, tumor proliferation, invasion and migration (13-16). Furthermore, in patients with high-risk NB, small nucleolar RNA host gene 1 is highly expressed and is strongly correlated with MYCN amplification (17,18).

As a member of the small nucleolar RNA host gene family, small nucleolar RNA host gene 16 (SNHG16) is highly expressed in several types of cancer (18-21). It is regulated by the Wnt pathway in colorectal cancer and induced breast cancer cell migration $(19,20)$. Although this suggests that SNHG16 may function as an oncogene in cancer, its underlying molecular mechanisms are unclear, particularly in pediatric NB. Therefore, the present study further investigated the effects of SNHG16 on NB.

\section{Materials and methods}

Clinical patients. All patients with NB (aged between 7 months and 8 years) were clinically and histopathologically diagnosed at Beijing Children's Hospital between May 2015 and December 2016 based on the International Neuroblastoma Staging System (INSS) for clinical staging of NB (22). The present study was approved by the Ethics Committees of Beijing Children's Hospital. A total of 40 surgical specimens were immediately snap-frozen in liquid nitrogen prior to total RNA extraction.

Cell culture and transfection. The NB cell line SH-SY5Y (\#CRL-2266; MYCN non-amplified) was obtained from the American TypeCulture Collection(ATCC); this cell line is widely used in mechanistic and drug development studies regarding NB $(23,24)$. Cells were cultured in Dulbecco's modified Eagle's medium (Corning, Inc.) supplemented with $10 \%$ fetal bovine serum (FBS; Corning, Inc.) in a humidified incubator containing $5 \% \mathrm{CO}_{2}$ at $37^{\circ} \mathrm{C}$. According to the manufacturer's protocol, synthetic small interfering (si)RNAs were transfected into cells at $\sim 50 \%$ confluence using the Lipofectamine RNAiMAX kit (Invitrogen; Thermo Fisher Scientific, Inc.). Cells were further analyzed $8 \mathrm{~h}$ post-transfection.

RNA interference. SH-SY5Y cells in the exponential growth phase were seeded for $24 \mathrm{~h}$ and were then transfected with $100 \mathrm{nM}$ siRNA at room temperature using Lipofectamine RNAiMAX (Invitrogen; Thermo Fisher Scientific, Inc.). siRNA oligonucleotides were synthesized by Sangon Biotech Co., Ltd., as follows: SNHG16 (siRNA1-SNHG16, 5'-CAGCA GUUGAGGGUUUGCUGUGUAUdTdT-3', siRNA2-SNHG16, 5'-GGACAACCUAGCUGUUGAAdTdT-3'); non-targeting control (5'-UUCUCCGAACGUGUCACGUTT-3'). Cells were returned to the incubator and were refreshed with normal medium $8 \mathrm{~h}$ post-transfection, and further experiments were performed at scheduled times.

$R N A$ extraction and reverse transcription-quantitative $P C R$ $(R T-q P C R)$. A total of $3 \times 10^{5} \mathrm{SH}-\mathrm{SY} 5 \mathrm{Y}$ cells/well were seeded in 6-well plates at $\sim 50 \%$ confluence and transfected with siRNA for $72 \mathrm{~h}$. Tumor tissues and cells were homogenized in TRIzol $^{\circledR}$ reagent (Invitrogen; Thermo Fisher Scientific, Inc.), and total RNA was extracted using the Direct-zol ${ }^{\mathrm{TM}}$ RNA Miniprep kit (Zymo Research Corp.). The RevertAid ${ }^{\mathrm{TM}} \mathrm{H}$ Minus First Strand cDNA Synthesis kit (Invitrogen) was used for RT, and cDNA templates were amplified with the SYBR Green Master mix (Applied Biosystems; Thermo Fisher Scientific, Inc.). Both RNA extraction and RT procedures were performed according to the manufacturers' protocols. qPCR thermal cycling was set as follows: Initial denaturation at $95^{\circ} \mathrm{C}$ for $10 \mathrm{~min}$; 40 cycles at $95^{\circ} \mathrm{C}$ for $15 \mathrm{sec}$ and $60^{\circ} \mathrm{C}$ for $60 \mathrm{sec}$; followed by melt curve at $95^{\circ} \mathrm{C}$ for $15 \mathrm{sec}, 60^{\circ} \mathrm{C}$ for $60 \mathrm{sec}$ and $95^{\circ} \mathrm{C}$ for $15 \mathrm{sec}$. For RNA expression detection, GAPDH was used as a reference gene. The primer sequences were as follows: SNHG16, forward 5'-CAGTCAGCCTCAGTTTCCAA-3', reverse 5'-AGGCAGGGCTGTGCTGAT-3'; and GAPDH, forward 5'-CGAGTCAACGGATTTGGTGGTAT-3' and reverse 5'-AGCCTTCTCCATGGTGAAGAC-3'. The relative fold-change in mRNA expression was calculated using the $2^{-\Delta \Delta \mathrm{Cq}}$ method (25).

Real-time cell proliferation assay. Cell proliferation was measured by xCELLigence real-time cell analysis (RTCA) (ACEA Biosciences, Inc.). Briefly, a total of $4 \times 10^{3} \mathrm{SH}-\mathrm{SY} 5 \mathrm{Y}$ cells were cultured in an adaptive E-plate (ACEA Biosciences, Inc.) and transfected with siRNA. The E-plate was then incubated at $37^{\circ} \mathrm{C}$ within the RTCA Station inside the incubator, and device-defined cell index values were recorded every $20 \mathrm{~min}$ for $72 \mathrm{~h}$. Increases in cell numbers altered the baseline impedance, which was monitored by gold micro-electrodes located at the bottom of the E-plate. Data analysis was performed using the RTCA Control Unit and preinstalled RTCA software 2.0 (ACEA Biosciences, Inc.).

Colony formation assay. A total of $2 \times 10^{3} \mathrm{SH}-\mathrm{SY} 5 \mathrm{Y}$ cells/well were seeded in 6-well plates and transfected with siRNA. After 14 days, cells were fixed with $4 \%$ paraformaldehyde for 10 min and stained with $0.1 \%$ crystal violet (Sigma-Aldrich; Merck $\mathrm{KGaA}$ ) for a further $10 \mathrm{~min}$ at room temperature. Images were captured using a light microscope (IX73; Olympus Corporation), in order to observe cell colony formation. Colonies containing $>50$ cells were counted and recorded for statistical analysis. Independent assays were conducted three times.

Wound healing assay. A total of $3 \times 10^{5} \mathrm{SH}-\mathrm{SY} 5 \mathrm{Y}$ cells/well were seeded in 6-well plates at $\sim 50 \%$ confluence, and a 1-ml micropipette tip was used to scratch the surface of the plate to create a 'wound'. After gentle washing with phosphate-buffered saline (PBS), the attached cells were transfected with siRNA. Wound images were captured under a light microscope (IX73; Olympus Corporation) at 0, 24, 48 and $72 \mathrm{~h}$. The wound 
width was measured and analyzed by AlphaView SA 3.4.0 software (ProteinSimple).

Transwell assay. Transwell chambers (pore size, $8 \mu \mathrm{m}$; Costar; Corning, Inc.) were used to conduct a migration assay. A total of $250 \mu \mathrm{l}$ serum-free medium containing $4 \times 10^{4}$ cells was added into the upper chamber, whereas $500 \mu \mathrm{l}$ complete medium was added into the bottom chamber. After $24 \mathrm{~h}$ at $37^{\circ} \mathrm{C}$, the cells on the upper chamber were discarded and the cells that had migrated to the lower surfaces of the filters were fixed with $4 \%$ paraformaldehyde for $10 \mathrm{~min}$ at room temperature. Images of the cells were captured using a light microscope (IX73; Olympus Corporation) following further staining with $0.1 \%$ crystal violet (Sigma-Aldrich; Merck KGaA) at room temperature for $10 \mathrm{~min}$.

Cell cycle analysis. A total of $3 \times 10^{5} \mathrm{SH}-\mathrm{SY} 5 \mathrm{Y}$ cells/well were cultured in 6-well plates; $72 \mathrm{~h}$ post-transfection, cells were trypsinized, centrifuged at $110 \mathrm{x}$ g for $5 \mathrm{~min}$, and resuspended in ice cold ethanol $(70 \%)$ at $4^{\circ} \mathrm{C}$ overnight. PBS containing $2 \% \mathrm{FBS}$ was added to spin down the cells at $440 \mathrm{x} \mathrm{g}$ for $5 \mathrm{~min}$. The cell pellet was then incubated in PBS containing $2 \% \mathrm{FBS}$, $10 \mu 11 \mathrm{mg} / \mathrm{ml}$ propidium iodide solution and $2 \mu 110 \mathrm{mg} / \mathrm{ml}$ RNAseA (Tiangen Biotech Co., Ltd.) for $30 \mathrm{~min}$ at $37^{\circ} \mathrm{C}$ in the dark. Cell cycle progression was assessed using a flow cytometer and CellQuest Pro 6.1 software (BD Biosciences); $\geq 1 \times 10^{4}$ cells were analyzed for each sample.

Acridine orange (AO)/ethidium bromide (EB) staining. $\mathrm{AO}$ and EB staining (Nanjing KeyGen Biotech Co., Ltd.) was used to visualize nuclear alterations characteristic of apoptosis. AO is a vital dye that stains live and dead cells, whereas EB only stains cells that have lost membrane integrity, thus indicating apoptosis. Live cells appear uniformly green, whereas apoptotic cells will incorporate $\mathrm{EB}$ and therefore will be stained red-orange with condensed nuclei (26). A total of $3 \times 10^{5} \mathrm{SH}-\mathrm{SY} 5 Y$ cells/well were cultured in 6-well plates, transfected with siRNA for $72 \mathrm{~h}$, and stained with $\mathrm{AO}(10 \mu \mathrm{g} / \mathrm{ml})$ and $\mathrm{EB}(10 \mu \mathrm{g} / \mathrm{ml})$ for $30 \mathrm{~min}$ at room temperature. Cellular apoptosis was subsequently viewed and images were captured under a fluorescence microscope (Olympus Corporation).

Caspase-3/7 activity detection. Caspase-3/7 activation was measured using the Caspase-Glo 3/7 assay (Promega Corporation) according to the manufacturer's protocol. Briefly, a total of $1 \times 10^{4}$ cells/well were seeded into 96 -well plates, transfected with siRNA, and cultured for $72 \mathrm{~h}$. Subsequently, $100 \mu \mathrm{l}$ Caspase-Glo 3/7 reagent was added to each well and the well contents were agitated on a plate shaker. Finally, samples were incubated at room temperature for $2 \mathrm{~h}$ and luminescence was measured using a SpectraMax Microplate Luminometer (Molecular Devices, LLC).

RNA-binding protein $(R B P)$ prediction analysis. Potential RBPs that bind SNHG16 (27) were systematically identified using starBase v2.0 software (starbase.sysu.edu.cn). The Cytoscape plug-in ClueGO was then used to identify Gene Ontology (GO) terms and interpret functions enriched for the predicted RBPs (28). Statistical parameters were set as follows: Right-sided hypergeometric test, $\mathrm{P}<0.05$ with Benjamini-Hochberg correction; GO levels, 6-14; Kappa score threshold, 0.4.
Table I. Clinical characteristics of patients with neuroblastoma enrolled in this study $(\mathrm{n}=40)$.

\begin{tabular}{lc} 
Parameter & Number of patie \\
\hline Age at diagnosis (years) & \\
$<1.5$ & $10(25)$ \\
$1.5-3$ & $7(17.5)$ \\
$3-7$ & $18(45)$ \\
$>7$ & $5(12.5)$ \\
Sex & \\
Male & $21(52.5)$ \\
Female & $19(47.5)$ \\
MYCN status & \\
Amplified & $8(20)$ \\
Non-amplified & $20(50)$ \\
Not clear & $12(30)$ \\
Tumor stage & \\
I & $3(7.5)$ \\
II & $7(17.5)$ \\
III & $13(32.5)$ \\
IV & $17(42.5)$
\end{tabular}

MYCN, MYCN proto-oncogene, bHLH transcription factor.

Statistical analysis. Publically available Gene Expression Omnibus (GEO) datasets (www.ncbi.nlm.nih.gov/geo) GSE62564 (29-32) and GSE16237 (33) were downloaded for SNHG16 expression analysis. Kaplan-Meier survival analysis and the log-rank test were performed based on survival times collected from the GSE62564 dataset. All data were analyzed using SPSS 19.0 software (IBM Corp.), and graphs were generated using GraphPad Prism 5.0 (GraphPad Software, Inc.). All results are expressed as the means \pm standard deviations. Multiple comparisons were assessed by one-way analysis of variance followed by Bonferroni post hoc test. Student's t-test was performed to analyze differences between two groups. $\mathrm{P}<0.05$ was considered to indicate a statistically significant difference.

\section{Results}

SNHG16 expression is positively associated with NB clinical characteristics. To explore the relationship between SNHG16 expression and the pathophysiological features of patients with NB, the GEO dataset GSE62564 (498 samples) was analyzed by stratification analysis based on INSS stage, risk group and MYCN status. SNHG16 was expressed at significantly higher levels in stage 4 tumors compared with in tumors at other stages (Fig. 1A). In addition, it was upregulated in high-risk NB and $M Y C N$ amplification subtypes compared with in low-risk NB (Fig. 1B) and $M Y C N$ non-amplification subtypes (Fig. 1C). Analysis of another independent dataset, GSE16237 (51 samples), confirmed these results (Fig. 1D and E). Analysis of NB tissue samples (Table I) revealed that SNHG16 expression was increased alongside clinical staging 
A

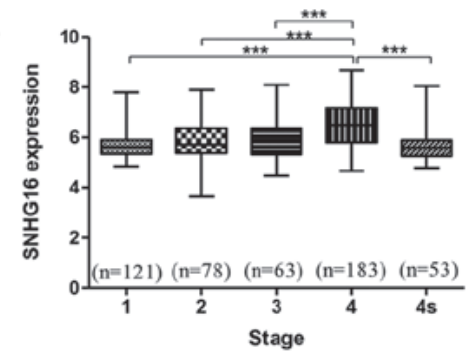

B

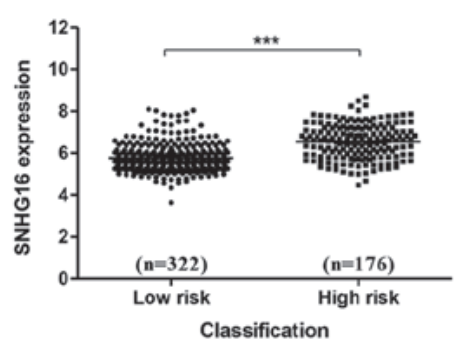

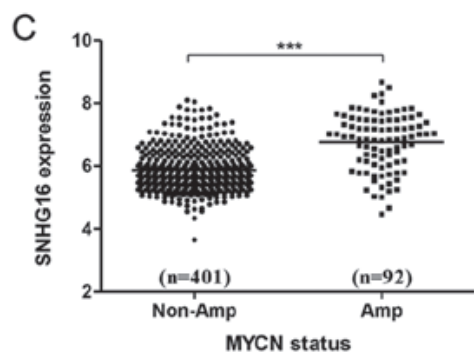
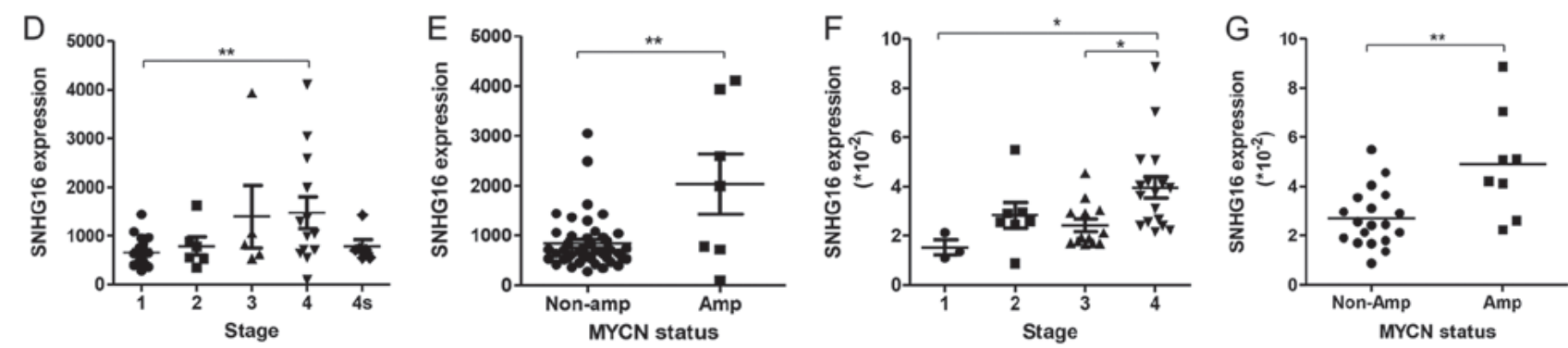

Figure 1. SNHG16 expression is positively associated with the clinical characteristics of NB. (A-C) $S N H G 16$ expression in samples stratified according to stage, risk group and $M Y C N$ status; data were obtained from the GSE62564 dataset. (D and E) SNHG16 expression according to stage and MYCN status; data were obtained from the GSE16237 dataset. (F and G) SNHG16 expression was measured by reverse transcription-quantitative PCR in collected NB samples. Data were obtained from three independent experiments. ${ }^{*} \mathrm{P}<0.05,{ }^{* *} \mathrm{P}<0.01,{ }^{* * *} \mathrm{P}<0.001$. MYCN, MYCN proto-oncogene, bHLH transcription factor; NB, neuroblastoma; SNHG16, small nucleolar RNA host gene 16.
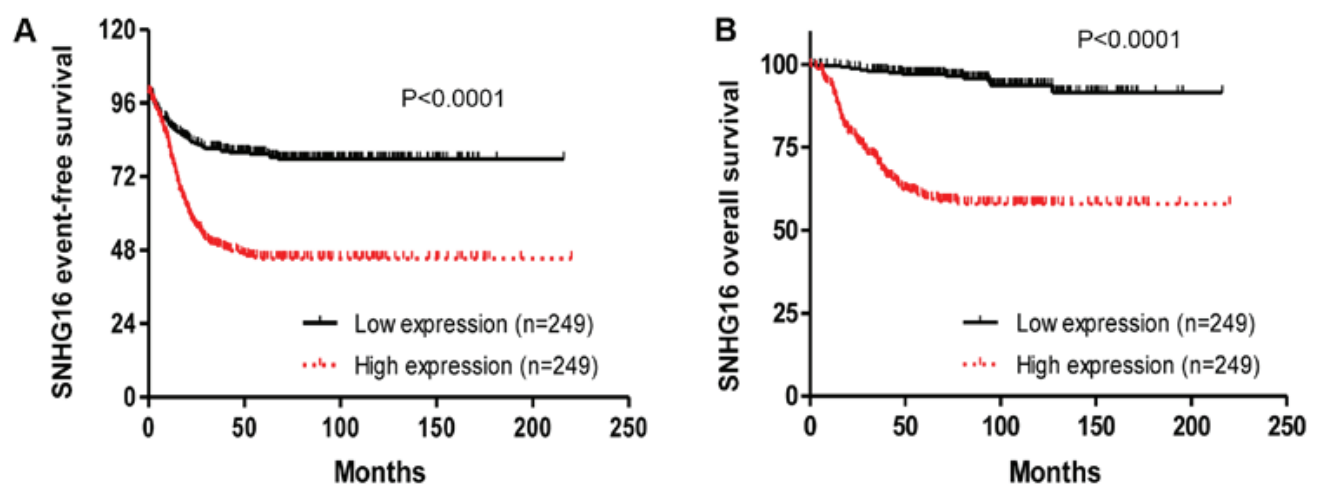

Figure 2. Kaplan-Meier survival analysis for patients with neuroblastoma in the GSE62564 dataset. Kaplan-Meier curves for (A) event-free survival and (B) overall survival of low-expression versus high-expression groups based on median SNHG16 expression. SNHG16, small nucleolar RNA host gene 16.

of NB tumor progression (Fig. 1F) and in $M Y C N$-amplified NB (Fig. 1G). These findings validated that SNHG16 expression was positively associated with NB progression.

To explore the prognostic value of $S N H G 16$, Kaplan-Meier survival analysis was conducted based on GSE62564 data. Patients with low or high expression of SNHG16 were grouped according to RNA sequencing data. Patients with high expression had worse event-free survival $(\mathrm{P}<0.0001)$ and overall survival $(\mathrm{P}<0.0001)$ than those with low expression (Fig. 2A and B), thus indicating that SNHG16 may be a prognostic marker for patients with NB.

SNHG16 silencing inhibits proliferation of SH-SY5Y cells. The gene knockdown efficiency of siRNAs was validated by RT-qPCR. siRNA1-SNHG16 and siRNA2-SNHG16 effectively silenced $S N H G 16$ expression (Fig. 3A). The real-time cell proliferation assay revealed that cell growth in the SNHG16 silencing group was significantly inhibited (Fig. 3B). This was confirmed by the colony formation assay, which demonstrated that $S N H G 16$ silencing markedly reduced colony formation in SH-SY5Y cells (Fig. 3C and D). These findings indicated that SNHG16 may contribute to NB cell proliferation. Both siRNA1 and siRNA2 exhibited effective knockdown efficiency, and were confirmed to inhibit cell proliferation; siRNA1 was used for subsequent experiments.

SNHG16 silencing inhibits migration of SH-SY5Y cells. To explore whether $S N H G 16$ affects cell migration, a wound healing assay was conducted using SH-SY5Y cells wounded with a micropipette tip. As shown in Fig. 4A, the wider width in the SNHG16 silencing group suggested that SNHG16 knockdown significantly suppressed the migratory ability of SH-SY5Y cells. Further quantification analysis revealed a significant difference in the width between the SNHG16 silencing group and the control group at 48 and $72 \mathrm{~h}$ after wounding (Fig. 4B). Furthermore, the results of a Transwell assay further indicated 
A



C

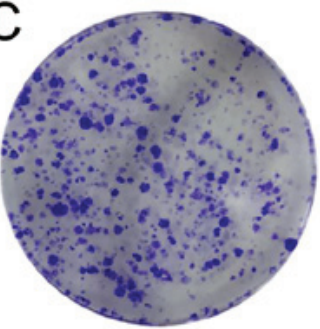

SiRNA-NC

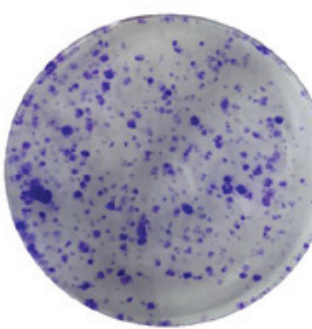

SiRNA1-SNHG16
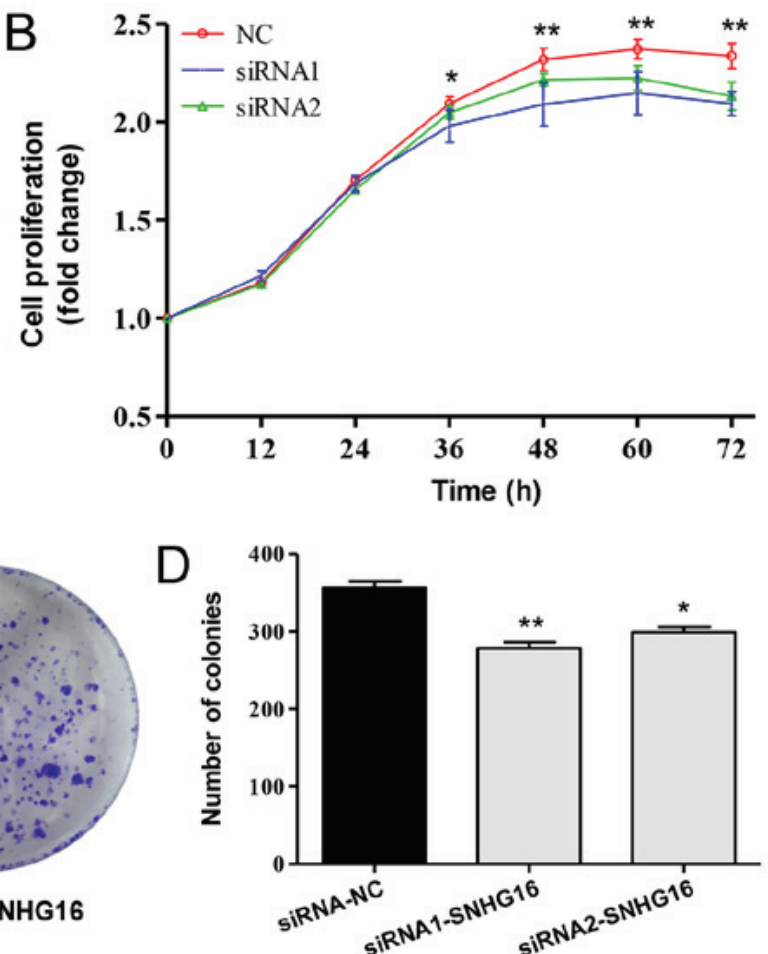

Figure 3. SNHG16 silencing inhibits cell proliferation in SH-SY5Y cells. (A) mRNA expression levels of SNHG16 in NC and siRNA-SNHG16 groups. (B) Real-time proliferation monitoring of SH-SY5Y cells. (C) Colony formation assay and (D) quantification analysis. Data were obtained from three independent experiments. ${ }^{*} \mathrm{P}<0.05,{ }^{* *} \mathrm{P}<0.01,{ }^{* * *} \mathrm{P}<0.001$ vs. the siRNA-NC group. NC, non-targeting control; siRNA, small interfering RNA; SNHG16, small nucleolar RNA host gene 16

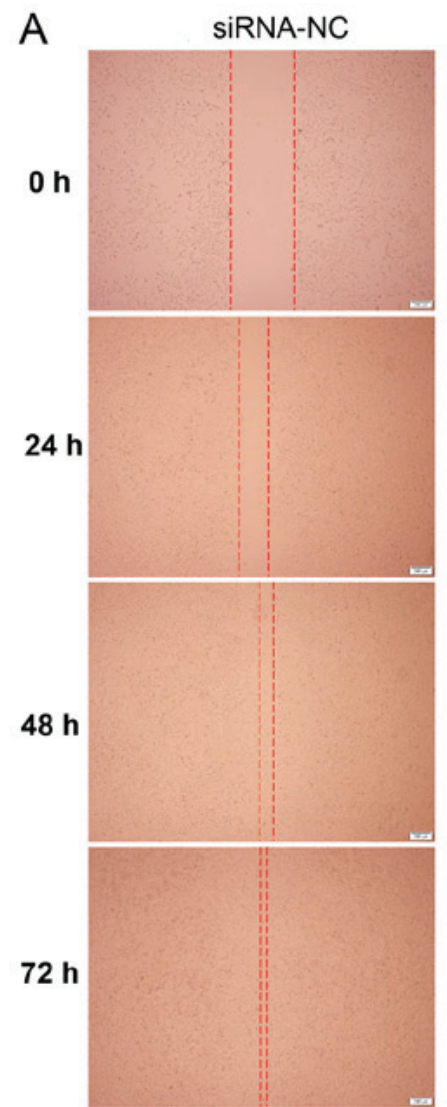

SiRNA1-SNHG16
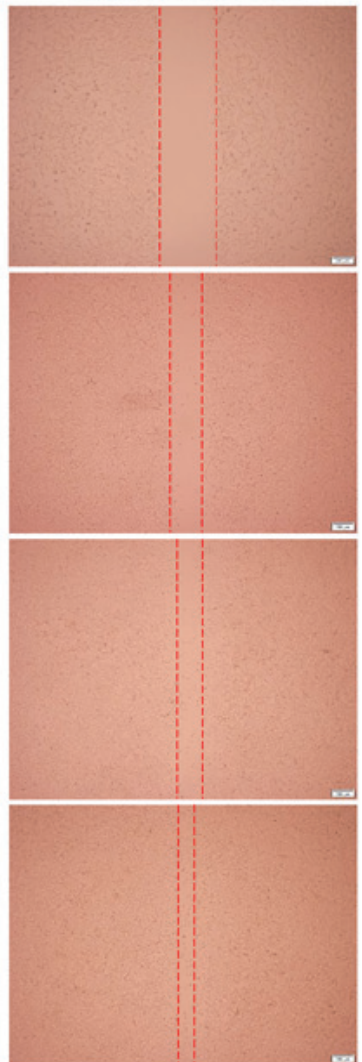

B

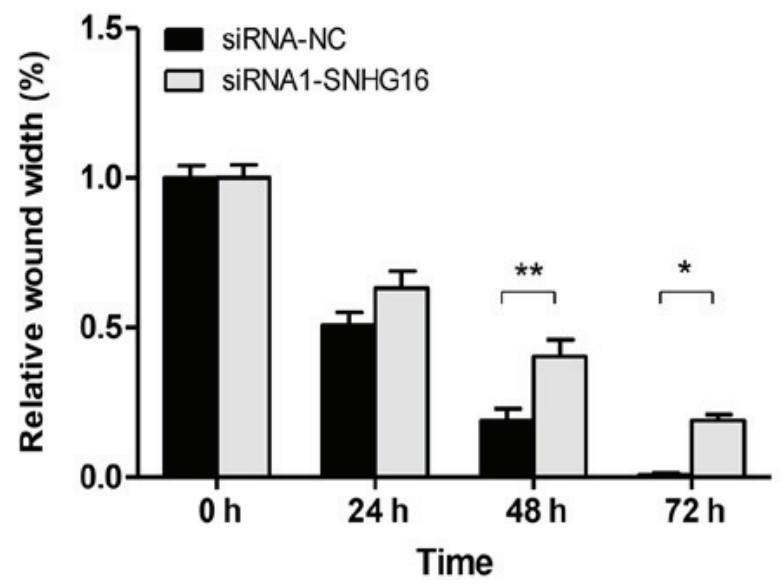

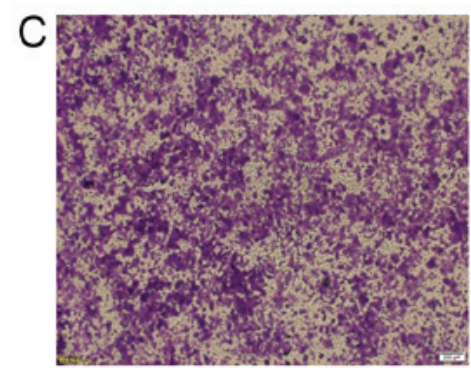

SiRNA-NC

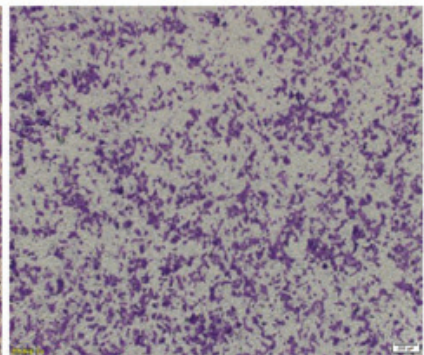

siRNA1-SNHG16

Figure 4. SNHG16 silencing inhibits migration of SH-SY5Y cells. (A) Representative wound healing images captured at 0,24 , 48 and 72 h after siRNA transfection. (B) Quantification analysis of wound width at the indicated time points. Data were from three independent experiments. (C) Transwell assay was performed to assess the migration of SH-SY5Y cells transfected with siRNAs. Scale bar, $200 \mu \mathrm{m} .{ }^{* *} \mathrm{P}<0.05,{ }^{* * *} \mathrm{P}<0.01$. NC, non-targeting control; siRNA, small interfering RNA; SNHG16, small nucleolar RNA host gene 16. 

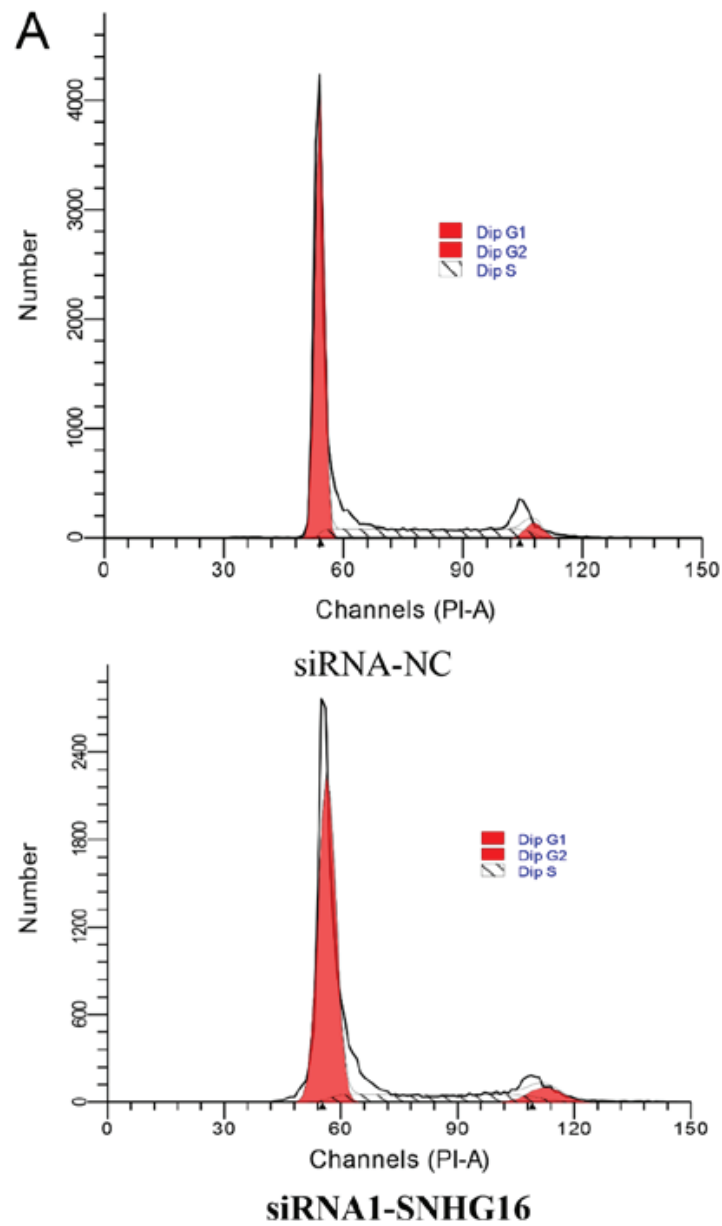

B

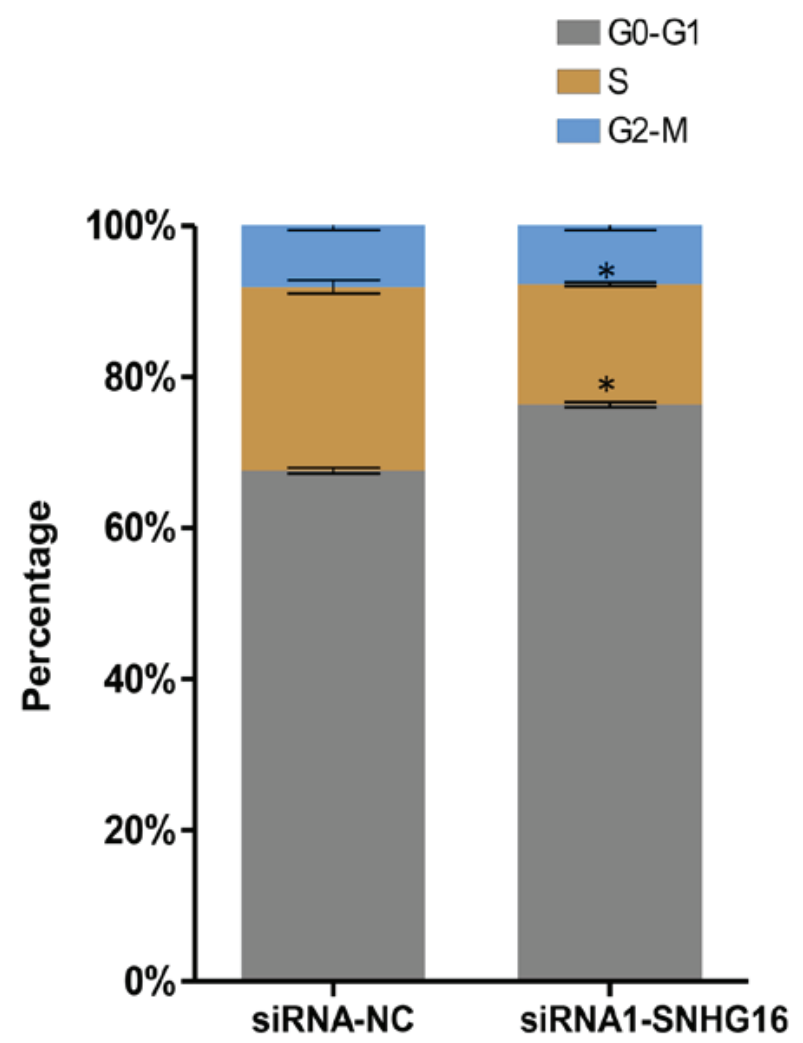

Figure 5. SNHG16 silencing induces cell cycle arrest in SH-SY5Y cells. (A) Flow cytometry was used to detect cell cycle alterations in SH-SY5Y cells after transfection with siRNA-NC or siRNA-SNHG16. (B) Percentage of cells in the $\mathrm{G}_{0} / \mathrm{G}_{1}$ phase was significantly increased in the siRNA-SNHG16 group compared with in the siRNA-NC group. Data were obtained from three independent experiments. ${ }^{*} \mathrm{P}<0.05$ vs. the siRNA-NC group. NC, non-targeting control; PI, propidium iodide; siRNA, small interfering RNA; SNHG16, small nucleolar RNA host gene 16.

that the migratory capacity of SH-SY5Y cells transfected with siRNA-SNHG16 was markedly decreased compared with the control group (Fig. 4C). These results suggested that SNHG16 knockdown inhibited migration of SH-SY5Y cells.

SNHG16 silencing induces cell cycle arrest in SH-SY5Y cells. The cell cycle is closely associated with cell proliferation; therefore, flow cytometry was used to investigate the potential effects of SNHGI6 on cell cycle alterations. The percentage of cells in $\mathrm{G}_{0} / \mathrm{G}_{1}$ phase was significantly increased, whereas the percentage in $\mathrm{S}$ phase was decreased in the SNHG16-knockdown group compared with in the control group (Fig. 5A and B; Table II). These results demonstrated that SNHG16 silencing in SH-SY5Y cells induced cell cycle arrest at the $G_{0} / G_{1}$ phase.

Apoptosis is undetectable in SH-SY5Y cells following SNHG16 silencing. Cellular apoptosis was measured by AO/EB staining following siRNA transfection. As shown in Fig. 6A, the cells in both groups appeared uniformly green with no distinct red-orange staining, indicating no detectable apoptotic cells. However, both cell number and density were reduced in the SNHG16-knockdown group, suggesting that SNHG16 silencing inhibited cell proliferation without apoptosis.
Table II. Cell cycle arrest of SH-SY5Y cells following siRNA-small nucleolar RNA host gene 16 transfection.

\begin{tabular}{llcc}
\hline & \multicolumn{3}{c}{ Distribution of cell cycle (\%) } \\
\cline { 2 - 4 } Group & $\mathrm{G}_{0} / \mathrm{G}_{1}$ & $\mathrm{~S}$ & $\mathrm{G}_{2} / \mathrm{M}$ \\
\hline $\mathrm{NC}$ & $67.60 \pm 0.65$ & $24.34 \pm 1.52$ & $8.08 \pm 0.97$ \\
siRNA & $76.35 \pm 0.59^{\mathrm{a}}$ & $15.94 \pm 0.41^{\mathrm{a}}$ & $7.72 \pm 0.98$
\end{tabular}

Data are expressed as the means \pm standard deviation from three independent experiments. ${ }^{\mathrm{a}} \mathrm{P}<0.05$. NC, negative control; siRNA, small interfering RNA.

Detection of caspase-3/7 activity also revealed that $S N H G 16$ knockdown had no significant effect on caspase-3/7 activity (Fig. 6B). These results indicated that SNHG16 knockdown did not induce apoptosis in NB.

Implied RNA-related mechanism of SNHG16 function. To explore the underlying molecular mechanism of SNHG16 function, starBase v2.0 was used to identify potential RBPs that bind SNHG16 (34). Predicted target proteins of SNHG16 
A
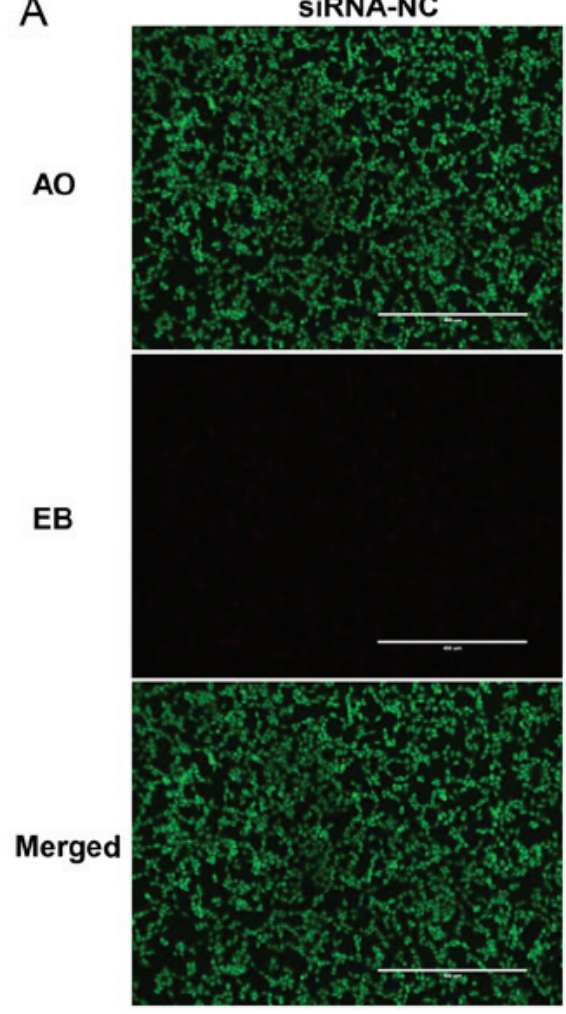
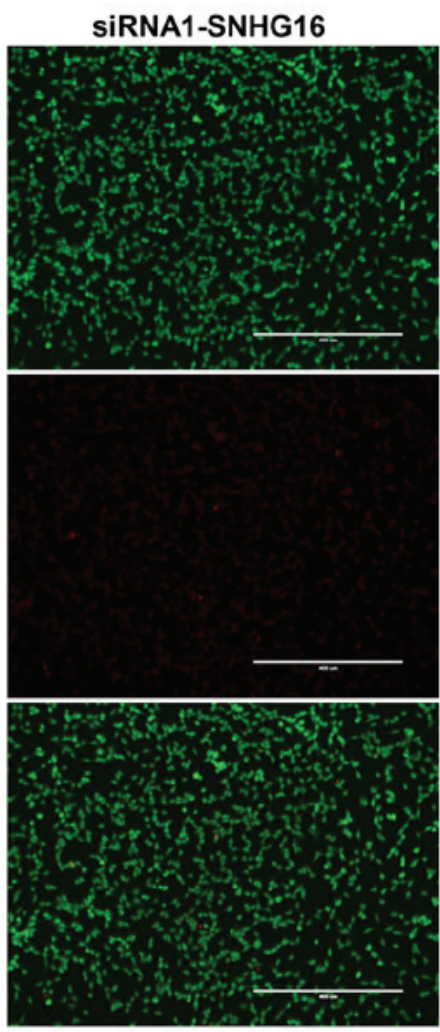

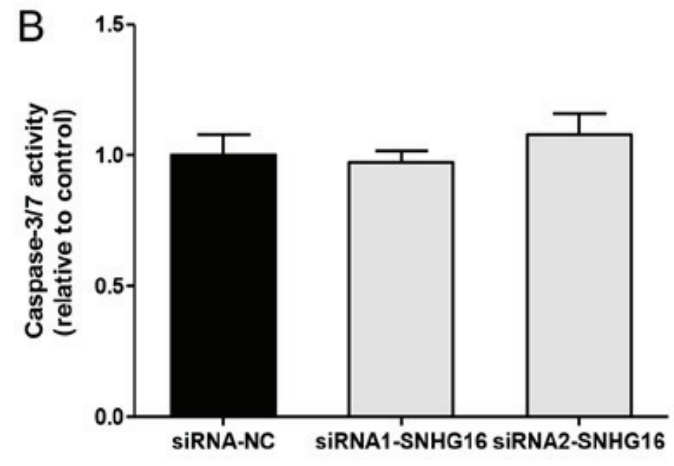

Figure 6. Apoptosis is undetectable in SH-SY5Y cells following SNHG16 silencing. (A) AO/EB staining revealed that cells in both groups appeared uniformly green without exhibiting distinct red-orange staining; however, cell number and density were reduced in the $S N H G 16$-knockdown group. (B) Caspase-3/7 activity was not significantly different between the siRNA-NC and SNHG16-knockdown groups. Data were normalized to the control and were obtained from three independent experiments. Scale bar, $400 \mu \mathrm{m}$. AO, acridine orange; EB, ethidium bromide; NC, non-targeting control; siRNA, small interfering RNA; SNHG16, small nucleolar RNA host gene 16.

are listed in Table SI, and functional enrichment analysis was conducted by Cytoscape. SNHG16-related RBPs were revealed to be mainly involved in the regulation of mRNA metabolic processes, gene silencing by miRNA, mRNA transport, RNA splicing and translation (Fig.7A-C), implying that the molecular mechanism underlying the effects of SNHG16 is associated with RNA-related processes.

\section{Discussion}

The results of the present study revealed that SNHG16 expression was associated with the clinical progression and prognosis of NB. In addition, SNHG16 silencing inhibited NB cell proliferation and migration, and induced cell cycle arrest at $\mathrm{G}_{0} / \mathrm{G}_{1}$ phase in SH-SY5Y cells.

lncRNAs have been reported to serve as signals of specific cellular states, and may identify cellular pathologies, provide prognostic value and predict therapeutic options for patients with cancer (35). The differential regulation of lncRNAs relative to coding genes may underlie the high degree of tissue-specific lncRNA expression. Furthermore, previous studies have suggested that the expression and dysregulation of IncRNAs are cancer type-specific compared with protein-coding genes $(36,37)$. This suggests that lncRNAs should be studied separately in different tumor types.

Sustaining proliferation and metastasis are the most fundamental features of tumors (38). The present results indicated that SNHG16 may contribute to NB progression.
Using GEO datasets, SNHG16 upregulation was revealed to be associated with clinical staging of $\mathrm{NB}$, and this was validated in a series of NB samples. SNHG16 knockdown significantly inhibited proliferation and migration of SH-SY5Y cells, whereas patients with high SNHG16 expression had poor overall survival. Previous studies have indicated that $S N H G 16$ promotes tumor proliferation via a diverse range of mechanisms. For example, SNHG16 contributes to cervical cancer by directly targeting miR-216-5p (39), and enhances tumor proliferation through epigenetically silencing $\mathrm{p} 21$, which acts as a tumor suppressor in bladder cancer $(21,40)$. SNHG16 has also been reported to be involved in the Wnt pathway in colorectal cancer and affects genes involved in lipid metabolism (19). Notably, lncRNAs have both positive and negative effects on NB proliferation. For example, high expression of lncRNAs, such as MYCN upstream transcript, account for NB tumorigenesis (38), whereas lincNeD-125 negatively controls NB proliferation and activates the anti-apoptotic factor BCL2 (15).

The primary aim of this study was to investigate the effects and underlying mechanism of SNHG16 in NB proliferation, and a series of experiments were performed. Notably, $M Y C N$ amplification is an independent marker for NB prognosis and risk stratification $(7,8)$. Existing evidence has suggested that SNHG16 is upregulated in a MYCN amplification cell line and in NB samples $(17,18)$. Therefore, MYCN amplification may affect $S N H G 16$ expression and subsequent function. In line with previous findings, the present results demonstrated that the expression of SNHG16 was increased in $M Y C N$-amplified 

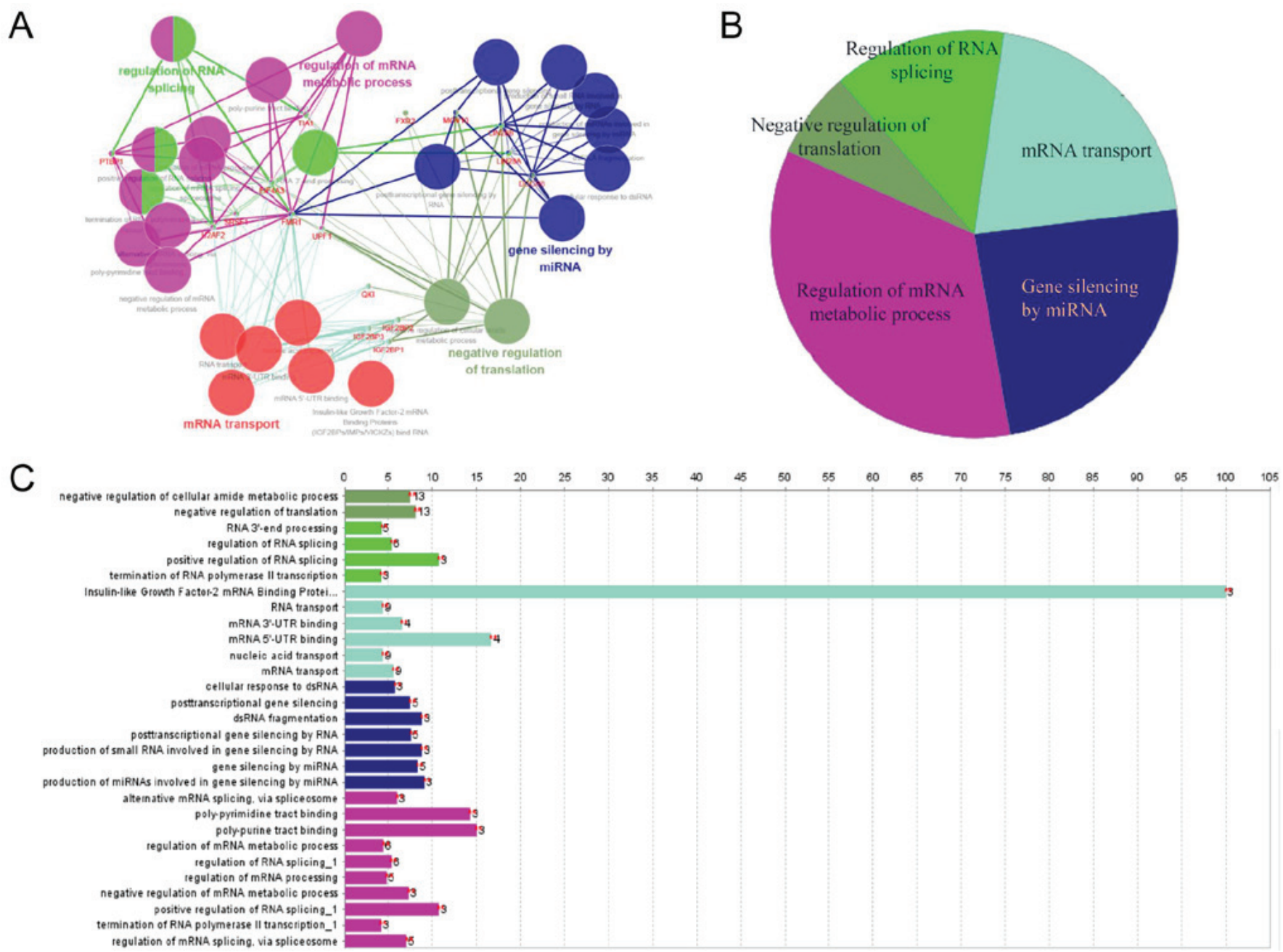

Figure 7. Functionally grouped Gene Ontology and pathway annotation of small nucleolar RNA host gene 16-related RBPs by ClueGO. (A) RBPs were functionally grouped and visualized in a network. (B) Overview chart presents functional groups for the RBPs. The name of the group is given by the group leading term (the most significant term in the group). (C) The chart presents the specific terms for the RBPs. The bars represent the number of genes from the analyzed cluster found to be associated with the term, and the label displayed on the bars is the percentage of found genes compared to all the genes associated with the term. RBP, RNA-binding protein.

NB samples compared with in $M Y C N$ non-amplified NB. A $M Y C N$ non-amplified cell line was selected to examine the role of SNHG16 in NB, because SH-SY5Y is a representative cell line of NB, according to the ATCC, and it has been widely used in mechanistic and drug development studies regarding NB $(23,24)$. The potential link between SNHG16 and MYCN amplification requires independent research for further clarification.

Various biological processes are associated with cell proliferation, including DNA damage, autophagy, apoptosis and the cell cycle (41). In the present study, SNHG16 silencing was revealed to affect cell cycle progression and lead to $\mathrm{G}_{0} / \mathrm{G}_{1}$ arrest in SH-SY5Y cells. This result supports a previous finding that $S N H G 16$ silencing is able to arrest gastric cancer cells at $\mathrm{G}_{0} / \mathrm{G}_{1}(42)$, as well as the reports that many lncRNAs are associated with $\mathrm{G}_{0} / \mathrm{G}_{1}$ block in various tumor types. For example, Yang et al (43) reported that hepatocellular carcinoma up-regulated EZH2-associated lncRNA is highly expressed in hepatitis B virus-related hepatocellular carcinoma and negatively regulates the expression of cyclin-dependent kinase inhibitors. Furthermore, Ye et al (44) reported that
lncRNA bladder cancer-associated transcript 1 silencing induces $G_{0} / G_{1}$ arrest through sponging miR-144. Therefore, cell cycle control may be a potential mechanism underlying SNHG16-mediated NB proliferation.

Sometimes cell cycle arrest is beneficial for DNA repair to maintain homeostasis; however, it can also cause cellular damage. Cells are equipped with cell cycle checkpoints to maintain genome stability; when cells have DNA damage to be repaired, or DNA replication is not complete, these checkpoints arrest the cell cycle $(45,46)$. This kind of cell cycle delay offers more time for the repair of DNA damage and cellular homeostasis. However, when cellular injuries are so severe that they exceed the cellular repair capacity, apoptosis may occur. Blocking the cell cycle is often associated with apoptosis; however, in this study, apoptosis was not detected by AO/EB staining and caspase-3/7 activity assay following SNHG16 silencing. This finding contradicts the previous findings of Christensen et al (19); this previous study reported that $S N H G 16$-induced apoptosis is dependent on caspase-3/7 activity in colorectal cancer cells. In addition, Lu et al (47) demonstrated that SNHG16 knockdown inhibits viability and induces apoptosis in glioma cells. Because 
IncRNA function is highly cancer type-specific compared with protein-coding genes $(48,49)$, this discrepancy may be accounted for by the different cell types or regulatory mechanisms between these studies and the present study.

The mechanisms underlying the effects of lncRNAs are highly extensive and complex, and may involve protein or DNA interactions (50). IncRNAs typically function through RBPs, including the polycomb-group proteins, heterochromatin protein 1 and DNA methyltransferases, whose expression fluctuates in tumor samples and therefore may provide prognostic clues (51). Chen et al (52) reported that the lncRNA $M A L A T 1$ regulates $\mathrm{p} 53$ through its interaction with cell cycle and apoptosis regulator 2, whereas RNA pulldown and RNA immunoprecipitation assays have demonstrated that the physical association of colon cancer-associated transcript 1 with Livin inhibits apoptosis in renal cell carcinoma (53). In the present study, bioinformatics analysis predicted various RBPs that may interact with $S N H G 16$, and functional enrichment analysis demonstrated that the potential RBPs regulated transcription and translation. However, to confirm these predictions, in-depth study and molecular experiments are urgently required. Nevertheless, the present results suggested that SNHG16 regulated cell proliferation, migration and the cell cycle in NB through multiple pathways associated with transcription and translation.

In conclusion, the present study demonstrated that high expression of SNHG16 was associated with NB progression and poor clinical outcome. SNHG16 silencing inhibited cell proliferation and migration, and induced cell cycle arrest at $\mathrm{G}_{0} / \mathrm{G}_{1}$. Bioinformatics analysis revealed that $\mathrm{SNHG16}$ regulated the proliferation of NB cells through multiple pathways associated with transcription and translation. Therefore, SNHG16 may serve as a molecular marker for NB therapy or prognosis.

\section{Acknowledgements}

The authors would like to thank Sarah Williams, PhD, for language editing a draft of this manuscript.

\section{Funding}

This work was supported by the National Natural Science Foundation of China (grant nos. 81702463 and 81702787) and the Beijing Health System Top Level Technical Personnel Training Plan (grant no. 20153079).

\section{Availability of data and materials}

All data generated or analyzed in this study are included in this published article.

\section{Authors' contributions}

$\mathrm{YYu}$ and $\mathrm{FC}$ performed most of the experiments and wrote the manuscript. YYa, YJ JS and JL performed some experiments. JT, SW and WY collected the clinical data and tissue samples. $\mathrm{PC}$ and $\mathrm{SH}$ performed the statistical analysis. YG, XN and HW designed the experiments and edited the manuscript. All authors have read and approved the final manuscript.

\section{Ethics approval and consent to participate}

This study was approved by the Ethics Committees of Beijing Children's Hospital, and followed the principles of the Helsinki Declaration II. Written informed consent was obtained from the legal guardians of the patients.

\section{Patient consent for publication}

The legal guardians of the patients involved in this study provided written informed consent prior to inclusion in the study.

\section{Competing interests}

The authors declare they have no competing interests.

\section{References}

1. Whittle SB, Smith V, Doherty E, Zhao S, McCarty S and Zage PE: Overview and recent advances in the treatment of neuroblastoma. Expert Rev Anticancer Ther 17: 369-386, 2017.

2. RogowitzE, Babiker HM, Kanaan M, Millius RA, Ringenberg QS and Bishop M: Neuroblastoma of the elderly, an oncologist's nightmare: Case presentation, literature review and SEER database analysis. Exp Hematol Oncol 3: 20, 2014.

3. Esiashvili N, Goodman M, Ward K, Marcus RB Jr and Johnstone PA: Neuroblastoma in adults: Incidence and survival analysis based on SEER data. Pediatr Blood Cancer 49: 41-46, 2007.

4. Maris JM,Hogarty MD, Bagatell R and Cohn SL: Neuroblastoma. Lancet 369: 2106-2120, 2007

5. Bosse KR and Maris JM: Advances in the translational genomics of neuroblastoma: From improving risk stratification and revealing novel biology to identifying actionable genomic alterations. Cancer 122: 20-33, 2016.

6. Salazar BM, Balczewski EA, Ung CY and Zhu S: Neuroblastoma, a paradigm for big data science in pediatric oncology. Int J Mol Sci 18: 18, 2016.

7. Schneiderman J, London WB, Brodeur GM, Castleberry RP, Look AT and Cohn SL: Clinical significance of MYCN amplification and ploidy in favorable-stage neuroblastoma: A report from the Children's Oncology Group. J Clin Oncol 26: 913-918, 2008.

8. London WB, Bagatell R, Weigel BJ, Fox E, Guo D, Van Ryn C, Naranjo A and Park JR: Historical time to disease progression and progression-free survival in patients with recurrent/refractory neuroblastoma treated in the modern era on Children's Oncology Group early-phase trials. Cancer 123: 4914-4923, 2017.

9. Peifer M, Hertwig F, Roels F, Dreidax D, Gartlgruber M, Menon R, Krämer A, Roncaioli JL, Sand F, Heuckmann JM, et al: Telomerase activation by genomic rearrangements in high-risk neuroblastoma. Nature 526: 700-704, 2015.

10. Quinn JJ and Chang HY: Unique features of long non-coding RNA biogenesis and function. Nat Rev Genet 17: 47-62, 2016.

11. Bhan A, Soleimani M and Mandal SS: Long noncoding RNA and cancer: A new paradigm. Cancer Res 77: 3965-3981, 2017.

12. Pandey GK and Kanduri C: Long noncoding RNAs and neuroblastoma. Oncotarget 6: 18265-18275, 2015 .

13. Bi S, Wang C, Li Y, Zhang W, Zhang J, Lv Z and Wang J: lncRNA-MALAT1-mediated Axl promotes cell invasion and migration in human neuroblastoma. Tumour Biol 39: 1010428317699796, 2017.

14. Yarmishyn AA, Batagov AO, Tan JZ, Sundaram GM, Sampath P, Kuznetsov VA and Kurochkin IV: HOXD-AS1 is a novel lncRNA encoded in HOXD cluster and a marker of neuroblastoma progression revealed via integrative analysis of noncoding transcriptome. BMC Genomics 15 (Suppl 9): S7, 2014.

15. Bevilacqua V, Gioia U, Di Carlo V, Tortorelli AF, Colombo T, Bozzoni I, Laneve P and Caffarelli E: Identification of linc-NeD125, a novel long non coding RNA that hosts miR-125b-1 and negatively controls proliferation of human neuroblastoma cells. RNA Biol 12: 1323-1337, 2015. 
16. Pandey GK, Mitra S, Subhash S, Hertwig F, Kanduri M, Mishra K, Fransson S, Ganeshram A, Mondal T, Bandaru S, et al: The risk-associated long noncoding RNA NBAT-1 controls neuroblastoma progression by regulating cell proliferation and neuronal differentiation. Cancer Cell 26: 722-737, 2014.

17. Sahu D, Hsu CL, Lin CC, Yang TW, Hsu WM, Ho SY, Juan HF and Huang HC: Co-expression analysis identifies long noncoding RNA SNHG1 as a novel predictor for event-free survival in neuroblastoma. Oncotarget 7: 58022-58037, 2016.

18. Yu M, Ohira M, Li Y, Niizuma H, Oo ML, Zhu Y, Ozaki T, Isogai E, Nakamura Y, Koda T, et al: High expression of ncRAN, a novel non-coding RNA mapped to chromosome 17q25.1, is associated with poor prognosis in neuroblastoma. Int J Oncol 34: 931-938, 2009.

19. Christensen LL, True K, Hamilton MP, Nielsen MM, Damas ND, Damgaard CK, Ongen H, Dermitzakis E, Bramsen JB, Pedersen JS, et al: SNHG16 is regulated by the Wnt pathway in colorectal cancer and affects genes involved in lipid metabolism. Mol Oncol 10: 1266-1282, 2016.

20. Cai C, Huo Q, Wang X, Chen B and Yang Q: SNHG16 contributes to breast cancer cell migration by competitively binding miR-98 with E2F5. Biochem Biophys Res Commun 485: 272-278, 2017.

21. Duan W, Du L, Jiang X, Wang R, Yan S, Xie Y, Yan K, Wang Q, Wang L, Zhang X, et al: Identification of a serum circulating IncRNA panel for the diagnosis and recurrence prediction of bladder cancer. Oncotarget 7: 78850-78858, 2016.

22. Brodeur GM, Pritchard J, Berthold F, Carlsen NL, Castel V, Castelberry RP, De Bernardi B, Evans AE, Favrot M and Hedborg F: Revisions of the international criteria for neuroblastoma diagnosis, staging, and response to treatment. J Clin Oncol 11: 1466-1477, 1993.

23. Li Z, Yan S, Attayan N, Ramalingam S and Thiele CJ: Combination of an allosteric Akt Inhibitor MK-2206 with etoposide or rapamycin enhances the antitumor growth effect in neuroblastoma. Clin Cancer Res 18: 3603-3615, 2012.

24. Radogna F, Cerella C, Gaigneaux A, Christov C, Dicato M and Diederich M: Cell type-dependent ROS and mitophagy response leads to apoptosis or necroptosis in neuroblastoma. Oncogene 35 3839-3853, 2016

25. Livak KJ and Schmittgen TD: Analysis of relative gene expression data using real-time quantitative PCR and the 2(-Delta Delta C(T)) method. Methods 25: 402-408, 2001.

26. Alarifi S, Ali D, Alkahtani S and Almeer RS: ROS-mediated apoptosis and genotoxicity induced by palladium nanoparticles in human skin malignant melanoma cells. Oxid Med Cell Longev 2017: 8439098, 2017.

27. Yang JH, Li JH, Shao P, Zhou H, Chen YQ and Qu LH: starBase: A database for exploring microRNA-mRNA interaction maps from Argonaute CLIP-Seq and Degradome-Seq data. Nucleic Acids Res 39 (Suppl 1): D202-D209, 2011.

28. Bindea G, Mlecnik B, Hackl H, Charoentong P, Tosolini M, Kirilovsky A, Fridman WH, Pagès F, Trajanoski Z and Galon J: ClueGO: A Cytoscape plug-in to decipher functionally grouped gene ontology and pathway annotation networks. Bioinformatics 25: 1091-1093, 2009.

29. Wang C, Gong B, Bushel PR, Thierry-Mieg J, Thierry-Mieg D, $\mathrm{Xu} J$, Fang H, Hong H, Shen J, Su Z, et al: The concordance between RNA-seq and microarray data depends on chemical treatment and transcript abundance. Nat Biotechnol 32: 926-932, 2014.

30. SEQC/MAQC-III Consortium: A comprehensive assessment of RNA-seq accuracy, reproducibility and information content by the Sequencing Quality Control Consortium. Nat Biotechnol 32: 903-914, 2014

31. Munro SA, Lund SP, Pine PS, Binder H, Clevert DA, Conesa A, Dopazo J, Fasold M, Hochreiter S, Hong H, et al: Assessing technical performance in differential gene expression experiments with external spike-in RNA control ratio mixtures. Nat Commun 5: 5125, 2014

32. Su Z, Fang H, Hong H, Shi L, Zhang W, Zhang W, Zhang Y, Dong Z, Lancashire LJ, Bessarabova M, et al: An investigation of biomarkers derived from legacy microarray data for their utility in the RNA-seq era. Genome Biol 15: 523, 2014.
33. Ohtaki M, Otani K, Hiyama K, Kamei N, Satoh K and Hiyama E: A robust method for estimating gene expression states using Affymetrix microarray probe level data. BMC Bioinformatics 11: $183,2010$.

34. Li JH, Liu S, Zhou H, Qu LH and Yang JH: starBase v2.0: Decoding miRNA-ceRNA, miRNA-ncRNA and protein-RNA interaction networks from large-scale CLIP-Seq data. Nucleic Acids Res 42D: D92-D97, 2014.

35. Wang KC and Chang HY: Molecular mechanisms of long noncoding RNAs. Mol Cell 43: 904-914, 2011.

36. Yan X, Hu Z, Feng Y, Hu X, Yuan J, Zhao SD, Zhang Y, Yang L, Shan W, He Q, et al: Comprehensive genomic characterization of long non-coding RNAs across human cancers. Cancer Cell 28: 529-540, 2015

37. Schmitt AM and Chang HY: Long noncoding RNAs in cancer pathways. Cancer Cell 29: 452-463, 2016.

38. Liu PY, Erriquez D, Marshall GM, Tee AE, Polly P, Wong M, Liu B, Bell JL, Zhang XD, Milazzo G, et al: Effects of a novel long noncoding RNA, IncUSMycN, on N-Myc expression and neuroblastoma progression. J Natl Cancer Inst 106: 106, 2014.

39. Zhu H, Zeng Y, Zhou CC and Ye W: SNHG16/miR-216-5p/ZEB1 signal pathway contributes to the tumorigenesis of cervical cancer cells. Arch Biochem Biophys 637: 1-8, 2018.

40. Cao X, Xu J and Yue D: lncRNA-SNHG16 predicts poor prognosis and promotes tumor proliferation through epigenetically silencing p21 in bladder cancer. Cancer Gene Ther 25: 10-17, 2018.

41. Hanahan D and Weinberg RA: Hallmarks of cancer: The next generation. Cell 144: 646-674, 2011.

42. Lian D, Amin B, Du D and Yan W: Enhanced expression of the long non-coding RNA SNHG16 contributes to gastric cancer progression and metastasis. Cancer Biomark 21: 151-160, 2017.

43. Yang F, Zhang L, Huo XS, Yuan JH, Xu D, Yuan SX, Zhu N, Zhou WP, Yang GS, Wang YZ, et al: Long noncoding RNA high expression in hepatocellular carcinoma facilitates tumor growth through enhancer of zeste homolog 2 in humans. Hepatology 54: 1679-1689, 2011

44. Ye JR, Liu L and Zheng F: Long noncoding RNA bladder cancer associated transcript 1 promotes the proliferation, migration, and invasion of nonsmall cell lung cancer through sponging miR-144. DNA Cell Biol 36: 845-852, 2017.

45. Malumbres M and Barbacid M: Cell cycle, CDKs and cancer: A changing paradigm. Nat Rev Cancer 9: 153-166, 2009.

46. Uryga A, Gray K and Bennett M: DNA damage and repair in vascular disease. Annu Rev Physiol 78: 45-66, 2016.

47. Lu YF, Cai XL, Li ZZ, Lv J, Xiang YA, Chen JJ, Chen WJ, Sun WY, Liu XM and Chen JB: IncRNA SNHG16 functions as an oncogene by sponging miR-4518 and up-regulating PRMT5 expression in glioma. Cell Physiol Biochem 45: 1975-1985, 2018.

48. Winkle M, Kluiver JL, Diepstra A and van den Berg A: Emerging roles for long noncoding RNAs in B-cell development and malignancy. Crit Rev Oncol Hematol 120: 77-85, 2017.

49. Chandra Gupta S and Nandan Tripathi Y: Potential of long non-coding RNAs in cancer patients: From biomarkers to therapeutic targets. Int J Cancer 140: 1955-1967, 2017.

50. Guttman M and Rinn JL: Modular regulatory principles of large non-coding RNAs. Nature 482: 339-346, 2012.

51. Ferrè F, Colantoni $A$ and Helmer-Citterich M: Revealing protein-IncRNA interaction. Brief Bioinform 17: 106-116, 2016.

52. Chen R, Liu Y, Zhuang H, Yang B, Hei K, Xiao M, Hou C, Gao H, Zhang X, Jia C, et al: Quantitative proteomics reveals that long non-coding RNA MALAT1 interacts with DBC1 to regulate p53 acetylation. Nucleic Acids Res 45: 9947-9959, 2017.

53. Chen S, Ma P, Li B, Zhu D, Chen X, Xiang Y, Wang T, Ren X, Liu C and Jin X: lncRNA CCAT1 inhibits cell apoptosis of renal cell carcinoma through up-regulation of Livin protein. Mol Cell Biochem 434: 135-142, 2017.

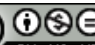

This work is licensed under a Creative Commons Attribution-NonCommercial-NoDerivatives 4.0 International (CC BY-NC-ND 4.0) License. 